\title{
The Challenge of Reducing Patient Falls in Hospitals
}

\author{
Cathy C. Schubert, MD \\ Indiana University School of Medicine and Richard L. Roudebush Veterans Affairs Medical Center \\ Indianapolis, Indiana
}

A fall is defined as an event resulting in a person coming to rest inadvertently on the ground or another lower level (1). Patient falls in health care settings are serious, widespread, and often complicated and multifactorial. Rates in U.S. hospitals range from 3.3 to 11.5 falls per 1000 patient-days (2). Patient death or serious injury associated with a fall while in a health care setting is considered a "never event" by the National Quality Forum and the Joint Commission, and in 2008, the Centers for Medicare \& Medicaid Services stopped reimbursing hospitals for treatment of injuries resulting from a fall while in the care of that facility, with many private insurers following suit (3).

One intervention often used by hospitals to prevent falls is the bedside "sitter," a nurse or other staff member who monitors the patient constantly and remains nearby at all times. Often, the patients needing sitters are older adults who have underlying cognitive concerns, such as dementia or delirium, and they need supervision and frequent reminders to follow safety instructions (such as pushing the call button for assistance before getting out of bed) when they are acutely ill and in an unfamiliar hospital environment. The theory behind sitters is that their presence at the bedside to immediately assist the patient will help prevent falls. Although this is a commonsense concept that is widely used in health care, sitters are expensive for hospital systems from both a financial and a staffing burden perspective.

Greeley and colleagues conducted a systematic review of the evidence of the effect of sitters and alternatives to them (such as video monitoring, "close observation units," and nurse assessment tools) on falls in adult patients admitted to medical/surgical or psychiatric wards of acute care hospitals (4). Studies could be randomized trials, quasi-experimental studies, time-series studies, or pre-post studies, and they had to include "one-to-one sitters" or a close observation unit and report falls as a primary outcome. Of the initial 4377 citations, only 20 studies met these eligibility criteria. No studies were deemed to have low risk of bias, and all 20 had at least 1 important methodological limitation, such as not defining a "fall" or not blinding the staff documenting the falls to the presence or absence of the intervention. Only 2 studies compared sitters with usual care, and their results conflicted in terms of the effect on fall rates. The remaining 18 studies evaluated alternatives to sitters. From these, the investigators found moderate-certainty evidence that interventions that included video monitoring decreased sitter use and either did not affect or decreased falls, but they found little convincing evidence that close observation units or nurse assessment tools were effective alternatives.

As a geriatrician with an inpatient consultation practice, I have long considered sitters to be one of my backbone recommendations for safety when caring for hospitalized older adults with frailty or cognitive issues. As noted by the review authors, "the rationale is intuitive and rooted in tradition," so it was

This is the author's manuscript of the work published in final form as:

Schubert, C. C. (2020). The Challenge of Reducing Patient Falls in Hospitals. Annals of Internal Medicine, 172(5), 356-357. https://doi.org/10.7326/M19-4005 
disconcerting to see the small quantity and poor quality of the evidence base for sitters or alternatives to sitters for reducing falls. I agree with the authors that this shallow evidence base should not yet compel us to stop using sitters in clinical care, and their study highlights the need for additional research in this area. In the meantime, however, if falls resulting in serious injury are to remain a never event, and if sitters are both expensive and possibly of limited efficacy, it would behoove us to begin shifting the focus of our efforts and resources to identifying highly effective and lower-cost approaches to preventing falls and to making our health care facilities and treatments safer and more evidence-based on a broader scale.

The goals of hospitalization are 3-fold and at times seemingly competing, especially in the care of patients with high medical and psychosocial complexity: 1) treat the acute illness, 2) keep the patient safe, and 3) help the patient at least maintain or recover to the baseline functional level (5). In an effort to better accomplish these goals, the Institute for Healthcare Improvement and the John A. Hartford Foundation have partnered with the American Hospital Association and the Catholic Health Association of the United States to launch an initiative called "age-friendly health systems" (6). To be age-friendly, health systems must deliver care based on a framework of evidence-based elements called the "4Ms": what matters (providing care that aligns with the patient's goals and preferences), medication (using agents that are age-friendly and do not interfere with the other $3 \mathrm{Ms}$ ), mentation (to prevent or identify and safely manage delirium), and mobility (to ensure patients move safely on a regular basis to maintain their function). This framework helps us move from disease-focused care to patient-centered, goalbased care that directly serves the needs of patients, accepting the complexity of their health and structuring their care around the goal of maintaining function. In this case, the $4 \mathrm{Ms}$ mean we would stop considering falls never events and would instead focus on mobilizing our patients as safely as possible while minimizing treatments that may negatively affect their cognition and function. "Age-friendly" means health care that views everything we do through the lens of each patient's goals of care.

In summary, Greeley and colleagues' study highlights the paucity of evidence for the effectiveness of sitters and alternatives to sitters at preventing falls in acute care hospitals, and their suggestion that additional research be conducted, especially for alternatives that would cost less, is valid. However, for acute care hospitals to be truly effective at keeping patients safe from never events, such as falls, we need to broaden our horizons and work to make the entire health system more age-friendly.

Disclaimer: Dr. Schubert is employed by the Richard L. Roudebush Veterans Affairs Medical Center and Indiana University School of Medicine. The views expressed in this article are those of the author and do not reflect the position or policy of the Department of Veterans Affairs, the U.S. government, or Indiana University.

Disclosures: Disclosures can be viewed at www.acponline.org/authors/icmje/ConflictOfInterestForms.do?msNum=M19-4005.

Corresponding Author: Cathy C. Schubert, MD, 1001 West 10th Street 111, Indianapolis, IN 46202; email, caschube@iu.edu. 


\section{References}

1. World Health Organization. Falls. Geneva: World Health Organization; 2018. Accessed at www.who.int/news-room/fact-sheets/detail/falls on 20 December 2019.

2. Bouldin EL, Andresen EM, Dunton NE, et al. Falls among adult patients hospitalized in the United States: prevalence and trends. J Patient Saf. 2013;9:13-7. [PMID: 23143749] doi:10.1097/PTS.0b013e3182699b64

3. Agency for Healthcare Research and Quality. Patient Safety Primer: Never Events. Rockville, MD: Agency for Healthcare Research and Quality; 2019. Accessed at https://psnet.ahrq.gov/primer/neverevents on 20 December 2019.

4. Greeley AM, Tanner EP, Mak S, et al. Sitters as a patient safety strategy to reduce hospital falls. A systematic review. Ann Intern Med. 2020;172:317-24. doi:10.7326/M19-2628

5. Ganz DA, Huang C, Saliba D, et al. Preventing Falls in Hospitals: A Toolkit for Improving Quality of Care. AHRQ publication no. 13-0015-EF. (Prepared by RAND Corporation, Boston University School of Public Health, and ECRI Institute under contract no. HHSA290201000017I TO \#1.) Rockville, MD: Agency for Healthcare Research and Quality; 2013. Accessed at https://psnet.ahrq.gov/issue/preventing-fallshospitals-toolkit-improving-quality-care on 20 December 2019.

6. Institute for Healthcare Improvement. Age-Friendly Health Systems. Boston: Institute for Healthcare Improvement; 2019. Accessed at www.ihi.org/Engage/Initiatives/Age-Friendly-HealthSystems/Pages/default.aspx on 20 December 2019. 